\title{
NATIONAL INSTITUTES \\ OF BIOSCIENCE \\ JOURNAL
}

\section{Genome Editing and the Future of Farming}

Conference held September 6th, 2016 at The Roslin Institute, Edinburgh

\section{The genetic architecture of economically important traits provides major challenges for the implementation of gene editing in livestock}

\author{
Dirk Jan de Koning \\ Department of Animal Breeding and Genetics \\ Swedish University of Agricultural Sciences \\ Box 7023, 75007 Uppsala, Sweden \\ Corresponding author: \\ Dj.de-koning@slu.se

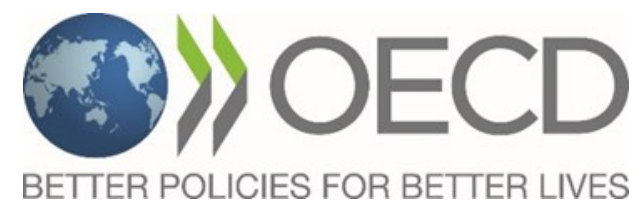

The opinions expressed and arguments employed in this publication are the sole responsibility of the authors and do not necessarily reflect those of the OECD or of the governments of its Member countries.

The Conference was sponsored by the OECD Co-operative Research Programme on Biological Resource Management for Sustainable Agricultural Systems, whose financial support made it possible for some of the invited speakers to participate in the Conference. 


\title{
The genetic architecture of economically important traits provides major challenges for the implementation of gene editing in livestock
}

\author{
Dirk Jan de Koning \\ Department of Animal Breeding and Genetics \\ Swedish University of Agricultural Sciences \\ Box 7023, 75007 Uppsala, Sweden \\ Corresponding author: \\ Dj.de-koning@slu.se
}

\begin{abstract}
Gene editing has been hyped as a game-changer in many biological fields including medicine and agriculture. This includes the potential to manipulate the DNA of livestock animals at sufficient throughput, both in terms of number of loci and animals, to consider gene editing as a routine component of livestock breeding programmes. In this article I will argue that the application of gene editing for complex traits in livestock will prove extremely challenging for a number of reasons: 1 ) our understanding of the genetic control of complex traits remains sketchy; 2) even with cutting edge 'omics technologies, the identification of functional mutations remains very challenging; 3 ) before selecting certain mutations for gene editing, we need to capture the pleiotropic effects of the mutation and test whether its effects are truly additive. With the current understanding of complex traits there is a risk that gene editing will revert to a candidate gene approach without knowledge or understanding of where the important mutations reside. This means that it will be some time before we can really benefit from gene editing for truly complex traits in livestock. In the meantime, gene editing could deliver quick wins by 'repairing' lethal recessive defects that are present in many elite breeding animals. I will also outline how gene editing can have an important role in the identification of QTN via in-vitro genetics.
\end{abstract}




\section{INTRODUCTION}

In a recent study (1) Jenko et al. introduce the concept of 'promotion of alleles by genome editing' (PAGE). Via extensive simulations they show that using gene editing to change the genotypes of a number of functional mutations (QTN) affecting a complex trait in a proportion of selection candidates can result in considerable genetic progress over and above 'standard' genomic selection. While the following sections will outline some difficulties in making this a reality, this is NOT a critique of the study Jenko et al., which introduces an innovative concept in a thorough and comprehensive manner. Neither is this article aiming to discourage the further development of gene editing approaches for livestock. Below, I will outline the challenges and risks of applying gene editing to engineer complex traits as well as suggest a 'low hanging fruit' alternative for the application of gene editing in livestock and ways in which gene editing can accelerate the discovery of functional mutations for complex traits.

\section{CHALLENGES FOR GENE EDITING TO ENGINEER COMPLEX TRAITS}

The main impediment for the successful implementation of gene editing for complex traits in livestock is that despite decades of genomics research our understanding of the genetic architecture of complex traits is still very sketchy. The Animal Quantitative Trait Loci (QTL) database (http://www.animalgenome.org/QTLdb) shows large number of QTL for the main livestock species: > 5000 QTL from 250 studies for chicken, > 16000 QTL from 557 studies in pig and > 80000 QTL from 710 studies in cattle (2). The number of QTL that has been resolved to the functional underlying mutation is still only a tiny fraction of this (3). A more recent boost to the detection of QTN, is the availability of large reference panels of animals with whole genome sequence data such as the 1,000 bulls genome consortium (4). The idea is that the whole genome sequence data includes all the functional mutations affecting the trait(s) of interest. Using imputation tools we can take the genotyping data from a genomewide association (GWAS) study and increase the marker density from the original single nucleotide polymorphisms (SNP) chip density (e.g. tens- or hundreds of thousands of SNPs) up to that of the whole genome sequence level (millions of SNPs)(5-7). Performing the GWAS on the imputed data should, in principle, allow the identification of the QTN among all the significant SNPs. Despite some successful applications of this approach (4), in many 
cases these studies identify a block of highly associated SNPs without identifying the QTN (810). There are a number of reasons why having (imputed) whole genome sequence information does not automatically facilitate the mapping of causal variants. 1) The reference genomes for the major livestock are still incomplete and contain unmapped regions and errors. 2) The annotation of livestock genomes is still very limited and a lot of functional regions remain to be identified and characterised. 3) The reference panels like the 1,000 bull genomes project represent a curated version of all the sequence variants, implying that a large number of rare variants may not make it into the reference panel. 4) Imputation is imperfect and has been shown to perform worse for lower minor allele frequencies (11). 5) Copy number variants (CNV) and other structural variants in the genomes are often omitted from imputation and/or ignored for association analyses. CNV have been shown to be associated with phenotypic variation in some studies, reviewed by (12). 6) Probably most important of all, the levels of linkage disequilibrium (LD) in livestock populations is the limiting factor for the resolution with which we can map QTL, regardless of the number of markers. One approach to reduce linkage disequilibrium around a QTL is to study the same QTL across breeds (9). However, even if the same QTL is detected across breeds, there may be no common haplotype around the QTL, suggesting multiple QTL alleles or multiple tightly linked loci (9). For successful identification of QTN, the availability of (imputed) whole genome sequence data is not sufficient. Additional bioinformatics and experimental filters, like eQTL experiments are often required to narrow down the putative QTN $(13,14)$.

Even if a QTN can be successfully identified, and confirmed, additional steps need to be taken before targeting it for gene editing. The pleiotropic effects on other traits need to be established to ensure that promoting this allele does not negatively affect other traits. If QTN with large effects are still segregating in a population undergoing artificial selection, it is important to evaluate whether the locus is under balancing selection because of pleiotropic effects. A good example is a $660 \mathrm{~Kb}$ deletion on BTA12 that has a beneficial effect on milk yield but is lethal in homozygous form (15). Furthermore, the gene action of the QTN needs to be validated to ensure that the effect is repeatable in subsequent generations and not constrained by epistatic interactions (16). 
Of course gene editing does not have to be restricted to identified QTN. One can, in principle, introduce mutations that have been identified in other breeds (like the hornless example in cattle (17)) or another species (like the influenza resistance in pigs (18)). With sufficient knowledge about the gene function, one can introduce mutations or deletions to change the functionality or expression level of a target gene. Given the limitations in our current understanding of the genetic control of complex traits, this approach will effectively be another incarnation of the candidate gene approach. Given the very limited success of this approach in identifying usable genetic variation for complex traits compared to whole genome approaches, it is unlikely that this will be a fruitful approach for the enhancement of economically important traits in livestock. Our research efforts should therefore focus on creating the tools and resources that are needed to advance our understanding of the genetics of complex traits like improved reference sequences, improved annotation of genomes and integrative tools to combine experimental results with public domain data to narrow down QTN candidates. In the meantime, considerable progress in complex traits can be made through genomic selection approaches that, while fit for use at present, would benefit from further refinement.

\section{LOW HANGING FRUIT AND ALTERNATIVE APPLICATIONS FOR GENE EDITING IN LIVESTOCK}

While the availability of high density SNP chips and reference panels with whole genome sequence information have not delivered many new QTN, they have facilitated the detection of recessive lethal mutations in livestock. With sufficiently large sample sizes, potentially in combination with a GWAS for fertility traits, the absence of homozygous individuals can be a clear indicator of a recessive lethal locus that is segregating in the population. This approach has identified multiple novel recessive lethal alleles in cattle populations $(4,15,19)$. These recessive lethal alleles impose restrictions on the breeding programme because matings between potential or known carriers should be avoided. Given the narrow genetic basis of the commercial dairy cattle breeds this further narrows the genepool and limits some of the genetic progress. A first target for gene editing in livestock could be to 'fix' recessive lethal mutations in elite germplasm, thus removing the constraints of mating carrier animals. This would not only be a quick gain for the breeding industry (breeding companies as well as farmers) it would also be one of the least controversial applications of gene editing as it is 
simply reverting to the 'wild type'. It will be interesting to investigate whether such gene editing approaches that are aimed at improving animal health/fertility are indeed more acceptable to the general public compared to those that are aimed at production increases.

While the identification of QTN is clearly a bottleneck in the implementation of gene editing for complex traits in livestock, gene editing itself can play a critical role in the discovery of QTN. Some years ago, we introduced the concept of in-vitro genetics for the improved detection of QTN (20). In brief, the starting point is a gene mapping study combined with an expression QTL study. That way we have the expression signature of the QTL: the genes that are differentially expressed between the two genotypes of the phenotypic QTL. The in-vitro part requires a relevant cell line in which the candidate mutation can be engineered. By comparing the overlap of the gene expression signature of the candidate mutation with that of the expression QTL, we can test if our candidate mutation represents the QTN (20). This concept was first demonstrated in-vivo for a QTN affecting bone strength in mice (13). Introducing gene edits in cell lines is an important component of gene editing techniques. The concept of in-vitro genetics requires routine availability of relevant cell lines and the ability to modify those for a range of candidate mutations. It is clear that the current developments in gene editing techniques in livestock can provide a real boost to in-vitro genetics approaches. I would envisage that the in-vitro genetics test becomes a routine component of the implementation pathway from gene mapping to gene editing for complex traits. Such a pathway is outlined in Figure 1. 


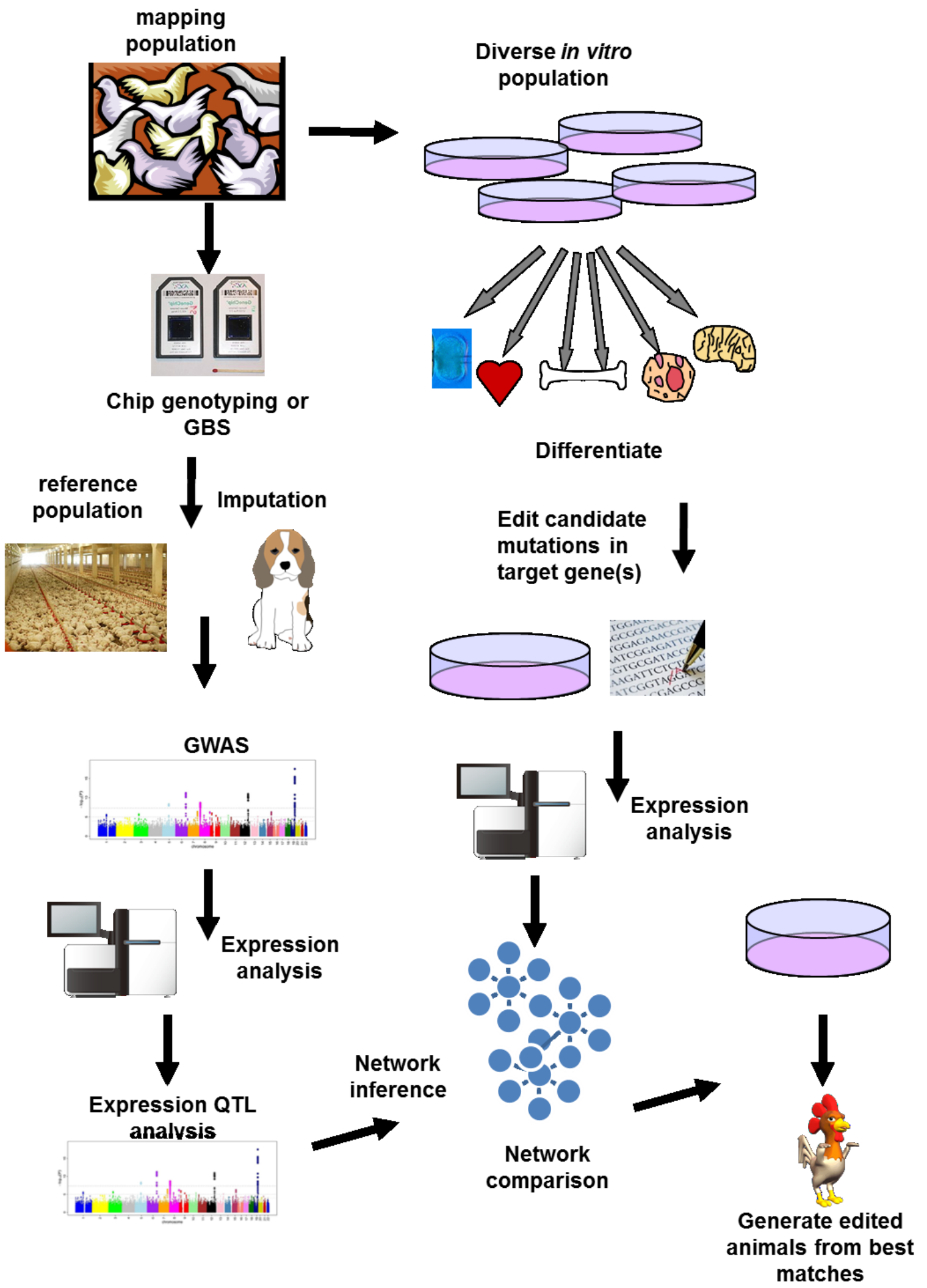

Figure 1. Schematic outline of how in-vitro genetics can be a routine component in the pathway from GWAS to gene editing for complex traits. A mapping population is used for GWAS and expression QTL mapping, potentially in combination with imputation for higher SNP density. Relevant cell lines are gene edited for candidate mutations and gene expression signatures are compared with that of the QTL. Cell lines with the best matching candidate mutations are developed to whole animals. 
In summary, I am optimistic and excited about the role that gene editing can play in livestock breeding, However, we need to be open about the challenges and prevent overpromising at a time when many of the techniques need a lot more development to be used routinely. While we need a certain measure of optimism to convince our funding bodies, we also need to make them aware of the limitations and the research funding required to deliver sustainable intensification of food production using the most appropriate biotechnology tools.

\section{ACKNOWLEDGEMENTS}

The author acknowledges colleagues at the Department of Animal Breeding and Genetics at SLU for valuabale discussions during the study day on gene editing. Further thanks are due to speakers and participants to the Mistra Biotech workshop on GMO livestock in 2014 as well as all contributors to this symposium on: Genome editing and the future of farming.

\section{FUNDING}

Funding for this contribution was provided by Mistra Biotech, a research programme financed by Mistra - The Swedish Foundation for Strategic Environmental Research and SLU.

\section{REFERENCES}

1. Jenko J, Gorjanc G, Cleveland MA, Varshney RK, Whitelaw CBA, Woolliams JA, et al. Potential of promotion of alleles by genome editing to improve quantitative traits in livestock breeding programs. Genet Sel Evol [Internet]. 2015 Dec [cited 2016 Nov 3];47(1). Available from: http://www.gsejournal.org/content/47/1/55

2. Hu Z-L, Park CA, Reecy JM. Developmental progress and current status of the Animal QTLdb. Nucleic Acids Res. 2016 Jan 4;44(D1):D827-33.

3. Weller JI, Ron M. Invited review: Quantitative trait nucleotide determination in the era of genomic selection. J Dairy Sci. 2011 Mar;94(3):1082-90.

4. Daetwyler HD, Capitan A, Pausch H, Stothard P, van Binsbergen R, Brøndum RF, et al. Whole-genome sequencing of 234 bulls facilitates mapping of monogenic and complex traits in cattle. Nat Genet. 2014 Jul 13;46(8):858-65.

5. Hickey JM, Kinghorn BP, Tier B, van der Werf JH, Cleveland MA. A phasing and imputation method for pedigreed populations that results in a single-stage genomic evaluation. Genet Sel Evol. 2012;44(1):9. 
6. Howie BN, Donnelly P, Marchini J. A Flexible and Accurate Genotype Imputation Method for the Next Generation of Genome-Wide Association Studies. Schork NJ, editor. PLoS Genet. 2009 Jun 19;5(6):e1000529.

7. Browning BL, Browning SR. Genotype Imputation with Millions of Reference Samples. Am J Hum Genet. 2016 Jan;98(1):116-26.

8. Mao X, Kadri NK, Thomasen JR, De Koning D, Sahana G, Guldbrandtsen B. Fine mapping of a calving QTL on Bos taurus autosome 18 in Holstein cattle. J Anim Breed Genet. 2015;

9. Mao X, Sahana G, De Koning D-J, Guldbrandtsen B. Genome-wide association studies of growth traits in three dairy cattle breeds using whole-genome sequence data. J Anim Sci. 2016;94(4):1426.

10. Duchemin SI, Glantz M, de Koning D-J, Paulsson M, Fikse WF. Identification of QTL on Chromosome 18 Associated with Non-Coagulating Milk in Swedish Red Cows. Front Genet [Internet]. 2016 Apr 15 [cited 2016 Nov 5];7. Available from: http://journal.frontiersin.org/Article/10.3389/fgene.2016.00057/abstract

11. Van Binsbergen R, Bink MC, Calus MP, van Eeuwijk FA, Hayes BJ, Hulsegge I, et al. Accuracy of imputation to whole-genome sequence data in Holstein Friesian cattle. Genet Sel Evol. 2014;46(1):41.

12. Clop A, Vidal O, Amills M. Copy number variation in the genomes of domestic animals. Anim Genet. 2012 Oct;43(5):503-17.

13. Mehrabian M, Allayee $H$, Stockton J, Lum PY, Drake TA, Castellani LW, et al. Integrating genotypic and expression data in a segregating mouse population to identify 5lipoxygenase as a susceptibility gene for obesity and bone traits. Nat Genet. 2005 Nov;37(11):1224-33.

14. Schadt EE, Lamb J, Yang X, Zhu J, Edwards S, GuhaThakurta D, et al. An integrative genomics approach to infer causal associations between gene expression and disease. Nat Genet. 2005 Jul;37(7):710-7.

15. Kadri NK, Sahana G, Charlier C, Iso-Touru T, Guldbrandtsen B, Karim L, et al. A 660-Kb Deletion with Antagonistic Effects on Fertility and Milk Production Segregates at High Frequency in Nordic Red Cattle: Additional Evidence for the Common Occurrence of Balancing Selection in Livestock. Leeb T, editor. PLoS Genet. 2014 Jan 2;10(1):e1004049.

16. Forsberg SKG, Bloom JS, Sadhu M, Kruglyak L, Carlborg Ö. Accounting for genetic interactions improves modeling of individual quantitative trait phenotypes in yeast [Internet]. 2016 Jun [cited 2016 Nov 5]. Report No.: biorxiv;059485v2. Available from: http://biorxiv.org/lookup/doi/10.1101/059485 
17. Carlson DF, Lancto CA, Zang B, Kim E-S, Walton M, Oldeschulte D, et al. Production of hornless dairy cattle from genome-edited cell lines. Nat Biotechnol. 2016 May $6 ; 34(5): 479-81$.

18. Palgrave CJ, Gilmour L, Lowden CS, Lillico SG, Mellencamp MA, Whitelaw CBA. SpeciesSpecific Variation in RELA Underlies Differences in NF- B Activity: a Potential Role in African Swine Fever Pathogenesis. J Virol. 2011 Jun 15;85(12):6008-14.

19. Sahana G, Nielsen US, Aamand GP, Lund MS, Guldbrandtsen B. Novel Harmful Recessive Haplotypes Identified for Fertility Traits in Nordic Holstein Cattle. Pas MF te., editor. PLOS ONE. 2013 Dec 20;8(12):e82909.

20. Haley CS, de Koning D-J. Towards in vitro genetics. TRENDS Genet. 2007;23(8):382-6. 